\title{
Usage du proche infrarouge pour la détermination de la qualité des graines oléagineuses
}

\author{
André MERRIEN \\ Mohammed KROUTI \\ José DIAZ

\section{CETIOM} \\ Laboratoire d'analyses, \\ 270, avenue de la Pomme-de-Pin, \\ BP 90635, \\ 45166 Olivet cedex, \\ France \\ $<$ merrien@cetiom.fr>
}

\begin{abstract}
The traceability requirements and allotment bring collectors or oilseed industry to seek for rapid, easy to implement, methods to determine the quality of productions. From this point of view, infrared methods offer possibilities. It's necessary however to be aware of both its advantages (fast, simple, multi-criteria non-destructive), and also limits: this indirect method requires constant validation by reference methods, and wide ranges of continuous and referenced values according to standards, effects "matrix" (eg Sunflower). Beyond the apprehension of some criteria on delivery (oil content), these methods are useful to breeders because they allow them a rapid screening of their equipment.
\end{abstract}

Key words: oilseed crops quality, infrared analysis
La mise en place de filières de qualité s'accompagne d'un effort important en matière de traçabilité, de respect des cahiers des charges mais aussi de contrôles analytiques. Si la mise en œuvre des méthodes de référence reste de la compétence des laboratoires, les acteurs des filières de qualité sont à la recherche de méthodes rapides, simples, peu onéreuses, qui pourront être mises en œuvre lors de la livraison au silo. De plus, la possibilité de cumuler sur le même équipement plusieurs calibrations (pack) offre l'avantage d'obtenir en un seul passage, plusieurs résultats.

Au niveau du sélectionneur, elle offre également I'avantage de ne pas être destructive. Au plan environnemental, $c^{\prime}$ est également un atout, puisque aucun solvant $n^{\prime}$ est requis.

L'objectif de disposer d'une méthode d'analyses rapide est multiple :

- rémunération de la qualité individualisée (teneur en huile de la graine de colza, teneur en protéines d'une graine de soja) ;

- allotement : il s'agit alors d'isoler telle ou telle production au regard d'une valorisation ultérieure et éviter le mélange de lots de qualités différentes : cela est particulièrement attendu dans le cas du tournesol oléique par exemple ; - screening à des fins de sélection sur des critères analytiques.

D'autres applications sont à l'étude: infraanalyseurs embarqués sur les moissonneuses batteuses et offrant la possibilité de cartographier la qualité de la récolte sur la parcelle, mise au point des paramètres du process indus- triel lors des étapes de la trituration (teneur en huile résiduelle des tourteaux par exemple). Pour atteindre ces objectifs, la mise en œuvre des techniques infrarouges est particulièrement adaptée, mais avec certaines limites inhérentes souvent à la matrice elle-même. Dans le cas des graines de tournesol par exemple, la structure physique de la coque et de l'amande rend quasiment impossible l'obtention de spectres sur graines entières ; il est alors nécessaire de broyer l'échantillon, ce qui augmente le temps de traitement pour obtenir le résultat et surtout, modifie le mode opératoire, et l'appareil doit être équipé d'un passeur de type « farine».

Dans tous les cas, l'élaboration d'une calibration nécessite une démarche expérimentale rigoureuse et devra être raccordée ainsi aux méthodes de référence qui feront toujours foi dans le cas des transactions commerciales. Ces déterminations indirectes sont principalement mises en œuvre dans une démarche comparative et constituent un réel outil d'aide à la décision.

\section{Principe}

Le principe de l'analyse par infrarouge repose sur l'absorption différentielle de la lumière dans le proche infrarouge (PIR) par la matière organique. Chaque liaison chimique, selon sa nature, oscille. Elle passe ainsi d'un état de base à un état d'excité lorsqu'elle absorbe l'énergie d'une source infrarouge dont la fréquence est égale à la fréquence de vibration de la liaison. Un spectromètre permet de mesurer ces variations.

L'analyse infrarouge est idéale pour détecter la présence de groupements fonctionnels.

On identifie des longueurs d'onde d'intérêt, c'est-à-dire des zones du spectre, l'on enregistrera une vibration qu'il sera ensuite possible de relier à une concentration de la molécule.

Le spectre d'absorption est complexe et chaque liaison chimique comporte plusieurs bandes. Dans le PIR, elles sont appelées harmoniques. Le PIR comporte les zones spectrales entre 800 et $2500 \mathrm{~nm}$. II correspond aux longueurs $d^{\prime}$ onde comprises entre le visible et l'infrarouge moyen. Selon le critère retenu et les caractéristiques (radicaux simples, chaînes hydrocarbonées, fonction azotée, simple liaison ou double liaison dans la molécule), différentes zones du spectre seront concernées. La force de liaison par exemple au sein d'une molécule va croissante au fur et à mesure que I'on passe de simples liaisons à des doubles ou à des triples (figure 1).

\section{Matériels disponibles}

Ce point est essentiel à prendre en compte lorsque l'on engage un travail sur les analyseurs infrarouges. Nous allons décrire sommairement les grandes catégories disponibles, aujourd'hui, sur le marché. Certains équipements fonctionnent par réflexion du rayonnement incident, et d'autres par transmittance : le rayonnement incident traverse alors la matrice analyseur infrarouge de type NIRS 


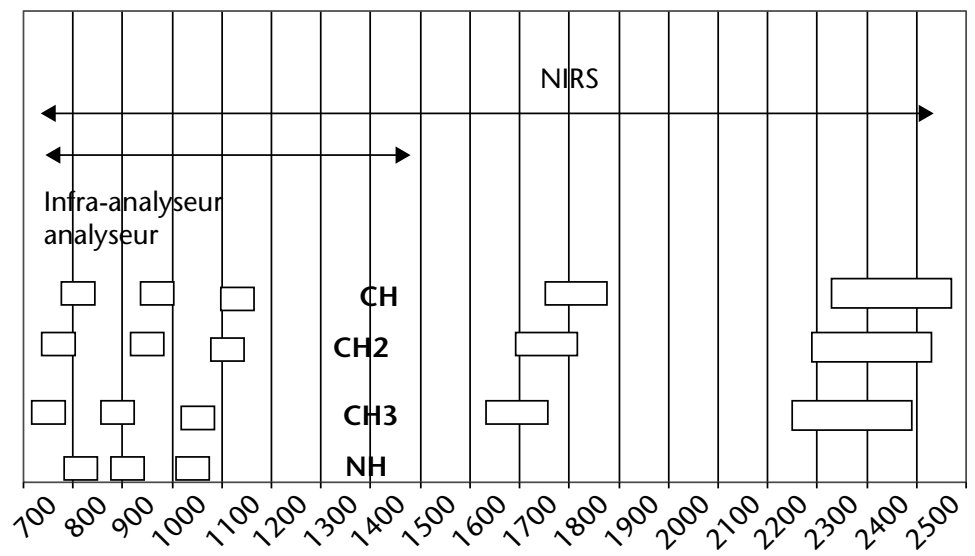

Longueur d'ondes (nm)

Figure 1. Principales bandes spectrales du proche infrarouge présentant un intérêt pour les liaisons $\mathrm{CH}, \mathrm{CH} 2$ et $\mathrm{CH} 3$ et NH (selon Bertrand D, Dufour E, 2000).

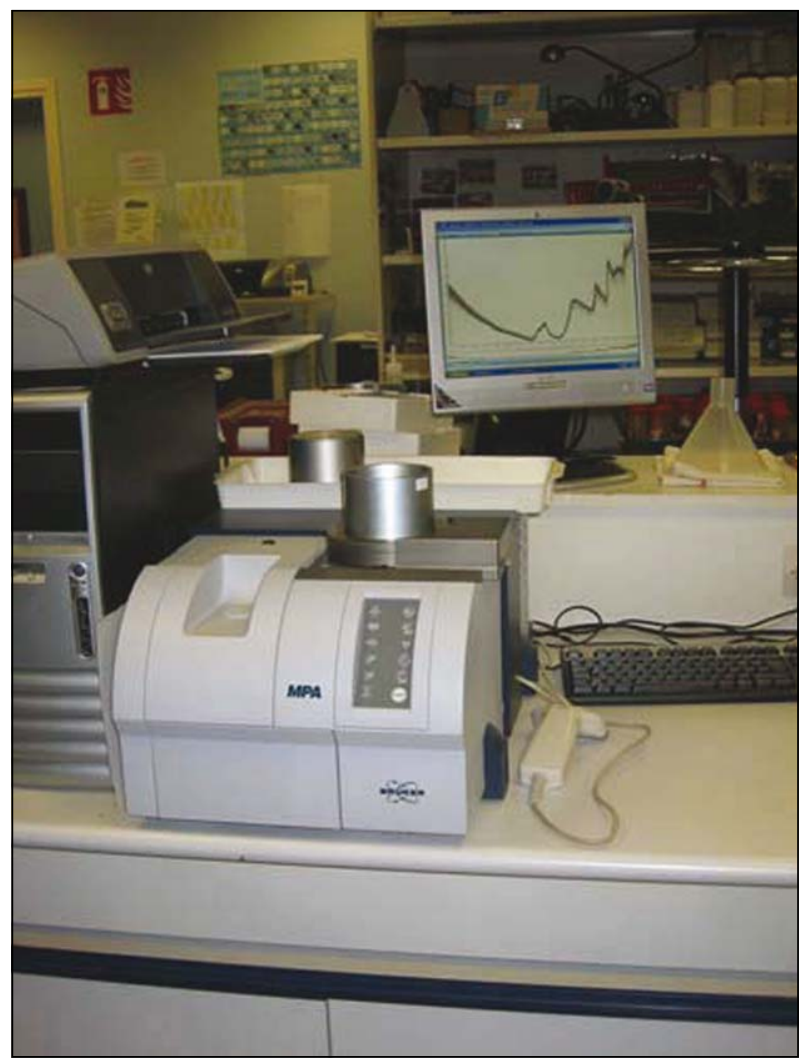

Figure 2. Exemple d'équipement NIRS (photo: M. Provot, Laboragro, InVivo).

(near infrared spectroscopy). Ce type d'équipement fonctionne par réflectance. On dispose, dans ce cas, d'un équipement qui permet d'analyser les déviations spectrales entre 700 et $2400 \mathrm{~nm}$ par la qualité de leur détecteur spectrométrique apte à analyser ces déviations. Grâce à cette amplitude de détection, ce type d'équipement couvrira une gamme d'application plus large. Toutefois, son prix est élevé, et il est principalement mis en œuvre au laboratoire ou dans les stations expérimentales (sélection, etc.). II nécessite par ailleurs des conditions stables (température, etc.) pour la bonne marche de l'équipement (figure 2).
Analyseur par transmittance (dit encore infraanalyseur ou Infratec ${ }^{\mathrm{TM}}$ ) : les performances du détecteur sont moindres : on couvre ici les bandes spectrales entre 850 et 1050 nm ; les critères analytiques sont en général simples (teneur en eau, teneur en huile, teneur en protéines). Ils sont faciles à mettre en œuvre et leur coût est abordable, rendant l'équipement adapté au niveau du silo (figure 3).

\section{Calibration}

II est nécessaire de disposer d'une gamme d'échantillons couvrant la variabilité la plus large possible concernant le paramètre recherché. On veillera à une bonne représentativité des échantillons entre années, variétés, origines géographiques. Ces échantillons seront caractérisés au plan analytique par les méthodes de référence. Si les premières calibrations sont réalisables avec une centaine d'échantillons, la validation de la calibration obtenue nécessite un second jeu de données indépendantes (figure 4).

De plus, il sera nécessaire d'en contrôler annuellement la performance par l'ajout d'une centaine de points chaque année.

Les spectres sont ensuite analysés par différentes méthodes statistiques allant des plus simples (régression linéaire multiple ou progressive) aux plus complexes (analyses neuronales).

Elles permettent de proposer des équations de calibration dont les paramètres statistiques essentiels seront l'écart-type de calibration (SEC) et la valeur prédictive du modèle (RMSEP). On admet, en général, qu'une calibration est valide lorsque la RMSEP n'est pas supérieure à 1,3 fois la SEC (figure 5).

\section{Quelles applications et leurs limites aujourd'hui pour les graines oléagineuses?}

\section{Teneur en huile}

Ce critère est sans nul doute le plus pertinent à prendre en compte dans les approches analytiques par infrarouge en raison de ses applications évidentes, notamment au niveau de la possible rémunération au producteur de la qualité de sa livraison.

\section{Colza}

Les calibrations existent et sont disponibles pour les principaux appareils (Infratec ${ }^{\mathrm{TM}}$ ou NIRS). Elles sont annuellement maintenues et commercialisées par Agroreso (www.agroreso. com) à partir d'une série d'échantillons dont les teneurs en huile sont établies au CETIOM selon les méthodes normalisées. Elles sont 


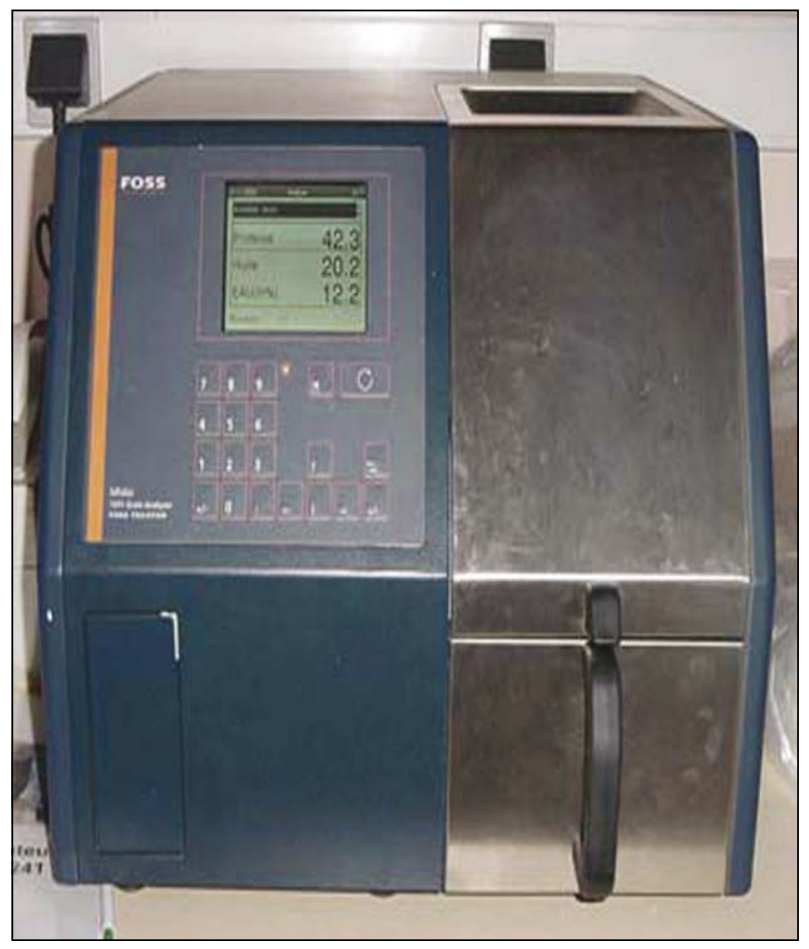

Figure 3. Exemple d'équipement infra-analyseur (photo : CETIOM).

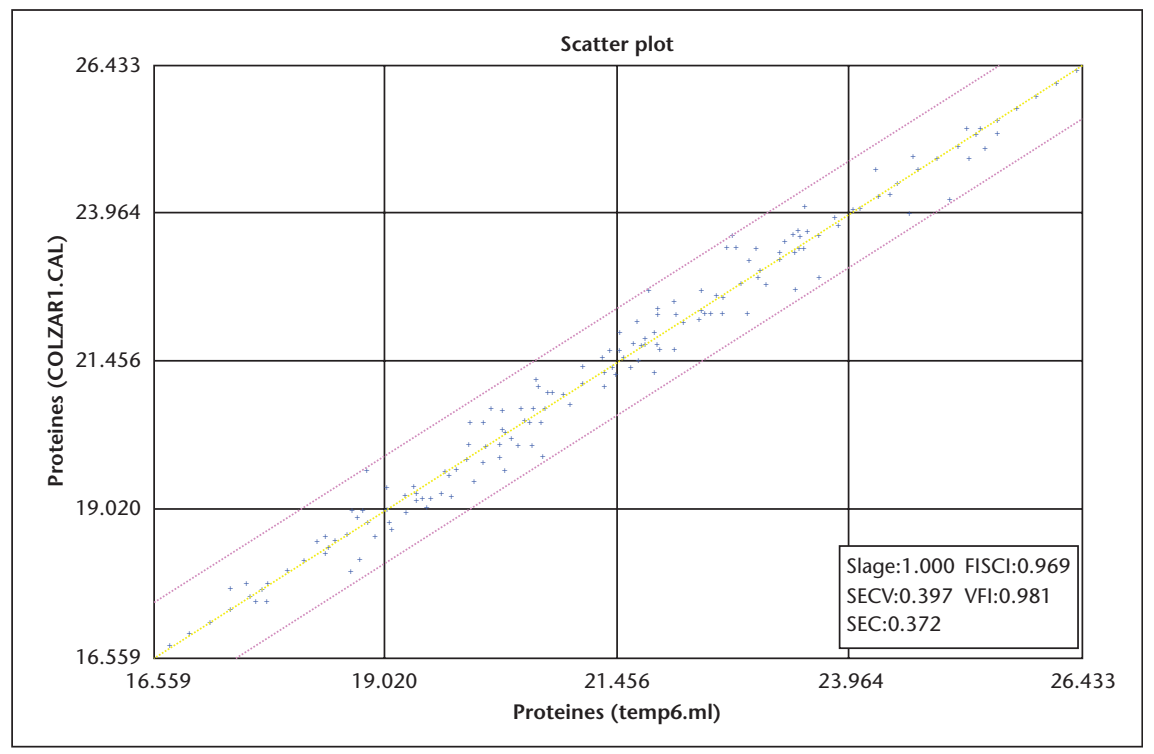

Figure 4. Relation entre la valeur prédite par la calibration IR et la valeur de référence pour la calibration « teneur en protéines » des graines de colza.

mises en œuvre principalement par les organismes stockeurs à la livraison des lots, mais aussi par les sélectionneurs qui travaillent sur ce critère.

\section{Tournesol}

Les calibrations sur graines entières sont loin d'être satisfaisantes en raison de la structure physique de la coque et de l'amande. Des

\section{Teneur en eau}

C'est sans nul doute le critère le plus facile à obtenir par cette technique sur Infratec ${ }^{\mathrm{TM}}$, les premières longueurs d'onde du spectre sont en effet discriminantes pour ce critère ; les calibrations sont régulièrement validées par le laboratoire du CETIOM qui possède, pour ce faire, une reconnaissance du Laboratoire national d'essai (LNE).

\section{Teneur en protéines}

À la différence des céréales, ce critère est moins important pour le colza et le tournesol, il le reste cependant pour le soja.

\section{Colza}

Les calibrations pour la détermination de la teneur en protéines des graines de colza sont aujourd'hui disponibles au CETIOM. Elles sont notamment mises en œuvre dans le cadre de l'évaluation des données du réseau variétés. Les performances sont maintenues chaque année.

\section{Tournesol}

Les efforts sont principalement concentrés, à ce jour, sur le paramètre « teneur en huile ».

\section{Soja}

Si la plupart des constructeurs offrent des calibrations pour l'analyse de la teneur en protéines des graines de soja, elles sont souvent le fruit de données obtenues outre-Atlantique. Le CETIOM a conduit pendant deux ans, avec les principaux constructeurs, un travail de validation de ces calibrations (et de nouveaux paramétrages) au regard des conditions de la production française. À ce jour, le travail se poursuit pour la surveillance annuelle de la calibration NIRS utilisée par les sélectionneurs (GIE Soja, Inra).

\section{Composition en acides gras de l'huile}

\section{Tournesol}

Les attentes sont extrêmement fortes pour les opérateurs des filières oléiques, de manière à pouvoir non seulement identifier des lots qui n'atteindraient pas la norme de contractualisation (généralement supérieure à $82 \%$ d'acide oléique), mais aussi de pouvoir faire un allotement dans le cas d'une production de tournesol à très haute teneur (>90\%) qui offre des débouchés plus attractifs lorsque préalablement contractualisés. À ce jour, les seules calibrations disponibles (avec des performances variables) le sont sur des équipements NIRS (Moschner et Korell, 2006; Gotor et al., 2008). Cela s'explique assez bien, puisque les zones spectrales d'intérêt sont plutôt au-dessus de 1500 nm, plage non explorée par les spectromètres équipant les Infratec ${ }^{\mathrm{TM}}$. 


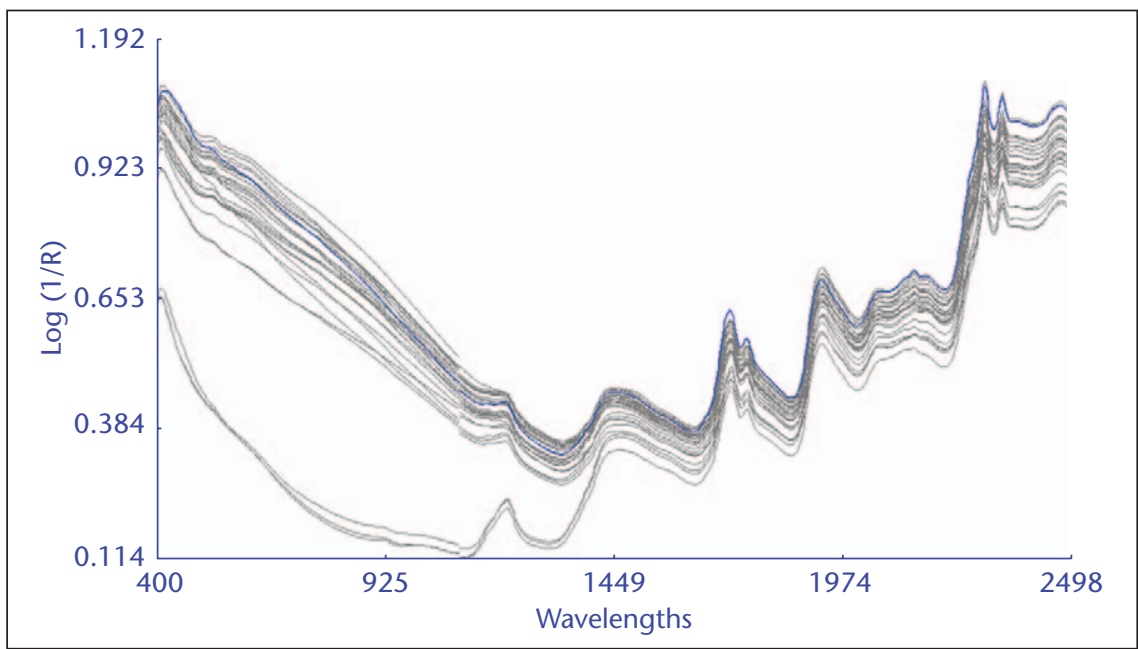

Figure 5. Exemple de famille de spectre obtenu au NIRS sur des graines de tournesol broyées.

\section{Colza}

Les calibrations sur NIRS sont plus faciles à obtenir et sont possibles sur des graines entières. Elles sont mises en œuvre principalement dans les programmes de sélection ; leur performance est adaptée pour un usage " en relatif », but recherché dans le cas de screening de matériel génétique. II n'existe pas, à ce jour, de calibrations sur ce critère mises au point sur des équipements Infratec ${ }^{\mathrm{TM}}$.

\section{Autres critères}

Parmi les autres critères visés par les techniques $d^{\prime}$ analyse infrarouge, on cite parfois la teneur en glucosinolates. À ce jour, les calibrations disponibles sont essentiellement mises au point en interne par les sélectionneurs en vue de contrôle des teneurs dans les graines. Les performances des calibrations produites restent toutefois insuffisantes.

D'autres critères (acidité) ont également fait I'objet de travaux (Lacoste, 2004).

\section{Conclusion}

Les techniques d'analyses rapides, principalement par infrarouge, offrent des perspectives intéressantes de par leur simplicité à être mises en œuvre et de par leur côté multicritère. Elles nécessitent toutefois une calibration rigoureuse qui doit être maintenue chaque année.

Il existe également des limites : certains critères resteront sans doute réservés aux méthodes de référence usuelles ou ne seront accessibles qu'avec les équipements les plus performants (acides gras chez le tournesol par exemple), mais aussi les plus onéreux.

Les applications les plus immédiates sont sans doute sur la teneur en huile des graines de colza : au plan génétique, elle a progressé ; la maîtrise de la fertilisation azotée (recherche de l'optimum) génère également entre 0,5 et 1 point d'huile de mieux.

Les techniques d'analyses rapides doivent permettre de prendre en compte la qualité des récoltes livrées et leur juste rémunération au regard de cette qualité : ce bonus est aujourd'hui mutualisé ; il est possible d'en assurer une individualisation, source de motivation et de progrès pour le producteur.

À côté des outils infrarouges, d'autres pistes mériteraient sans doute un peu d'investigation: les techniques d'analyse d'image par exemple permettraient la détermination rapide de taux d'impuretés.

\section{RÉFÉRENCES}

Bertrand D, Dufour E. La spectrométrie infrarouge et ses applications analytiques. Paris : Ed Lavoisier, 2000.
Gotor A, Moreau P, Gaillard A, Calmon A. Caractérisation par infrarouge des teneurs en acides gras de la graine entière décortiquée de tournesol. Proc. 17th Intern. Sunflower Conf. CordobaSpain, 2008.

Lacoste F. Détermination de l'acidité et de l'indice de peroxyde dans les huiles d'olive vierges et dans les huiles raffinées par spectrométrie proche infrarouge à transformée de Fourier. $\mathrm{OCL}$ $2004 ; 11: 70-5$.

Moschner C, Korell B. Estimating the content of free fatty acid in high oleic sunflower seeds by near infrared spectrometry. Eur J Lipid Sci Technol 2006 ; $108: 606-13$.

Perez-Vich B, Velasco L, Fernadez-Martinez J. Determination of seed oil content and fatty acid composition in sunflower through the analysis of intact seeds, husked seeds, meal and oil by near infrared reflectance spectrometry. JAOCS $1998 ; 75: 547-55$. 